\title{
Assessing the stability of biobank donor preferences regarding sample use: evidence supporting the value of dynamic consent
}

\author{
Joel E. Pacyna $\mathbb{1}^{1}$ - Jennifer B. McCormick ${ }^{2}$ Janet E. Olson $\mathbb{1}^{3}$ - Erin M. Winkler ${ }^{4}$ Josh T. Bublitz ${ }^{3}$. \\ Matthew A. Hathcock ${ }^{3} \cdot$ Richard R. Sharp $\mathbb{D i}^{1,3}$
}

Received: 10 December 2019 / Revised: 3 March 2020 / Accepted: 24 March 2020 / Published online: 23 April 2020

(c) The Author(s), under exclusive licence to European Society of Human Genetics 2020

\begin{abstract}
Dynamic consent has been proposed as a strategy for addressing the limitations of traditional, broad consent for biobank participation. Although the argument for dynamic consent has been made on theoretical grounds, empirical studies evaluating the potential utility of dynamic consent are needed to enhance deliberations about the merits of dynamic consent. Few studies have assessed such considerations as whether donor preferences may change over time or if participants would use a dynamic consent mechanism to modify preferences when they change. We administered a 66-item survey to participants in a large DNA biobank. The survey sought to gauge the stability of donor preferences specified at the time of biobank enrollment, specifically the stability of donors' preference regarding posthumous availability of biospecimens to next-of-kin. We received 1164 completed surveys for a response rate of $72 \%$. Forty percent of respondents indicated a preference regarding sample availability on the survey (T2) that was inconsistent with the preference they had expressed when they enrolled in the biobank (T1). Most (94\%) individuals with inconsistent preferences regarding sample availability had initially restricted sample availability at $\mathrm{T} 1$ but were comfortable with broader availability when asked at the time of the survey (T2). Our findings demonstrate that preferences regarding sample use expressed at the time of enrollment in a DNA biobank may not be reliable indicators of donor preferences over time. These findings lend empirical support to the case for a dynamic consent model in which biobank participants are approached over time to clarify their views regarding sample use.
\end{abstract}

\section{Introduction}

Dynamic consent has been proposed as a mechanism for respecting the interests and preferences of biobank donors $[1,2]$. Dynamic consent uses web-based interfaces to allow biobank donors to clarify their individual preferences regarding sample distribution and use. Dynamic consent could also provide a portal through which biobank donors are able to track which studies are utilizing their

Richard R. Sharp

sharp.richard@mayo.edu

1 Biomedical Ethics Research Program, Mayo Clinic, Rochester, MN, USA

2 Department of Humanities, Penn State College of Medicine, Hershey, PA, USA

3 Health Sciences Research, Mayo Clinic, Rochester, MN, USA

4 Center for Individualized Medicine, Mayo Clinic, Rochester, MN, USA biospecimens and health data, and serve as a mechanism for researchers to communicate results of specific studies back to donors [3].

Consideration of dynamic consent is timely as several new DNA biobanks, including the All of Us Research Program [4], are recruiting very large numbers of sample donors and are considering how best to manage these important collections over time. Although dynamic consent has been proposed as a strategy for addressing the limitations of traditional, broad consent for biobank participation [5-9], it is unclear whether biobank participants would have reasons to use a dynamic consent mechanism to adjust their sample use preferences. Little data exists to suggest whether biobank donors' sample use preferences evolve or remain stable after participants enroll in a DNA biobank. Although the argument for dynamic consent has been made on theoretical grounds, empirical studies evaluating the potential utility of dynamic consent are much needed to enhance deliberations about the merits of dynamic consent, especially given the costs of such a mechanism and the practical challenges of migrating existing large collections to a 
Fig. 1 Option to restrict posthumous availability to one's biospecimen from legal next-of-kin. This textbox with checkbox option was included in the biobank enrollment consent form. We included the same textbox option in the survey.

\author{
Since your samples contain your genetic information, your family may want access to \\ them after you die. They might use that information for many different things, such \\ as clarifying health risk in your family or determining if you were genetically related \\ to someone. It is possible that your samples will be used up during your lifetime and \\ will not be available to your family. \\ If you do not want your family to have access to your blood sample after you die, \\ please check the box below: \\ No, I do not want my family to have access to my sample after I die. \\ If you do not check the box, you are telling us that your legal next-of-kin has your \\ permission to test your sample in any way that is allowed by law. We cannot \\ absolutely guarantee that your next-of-kin will get access to your samples because \\ Mayo Clinic will need to comply with laws existing at that time.
}

dynamic consent platform. Before efforts are launched in support of implementing dynamic consent, data are needed to assess whether biobank donor preferences regarding sample use may change over time, which may be a predictor of whether biobank participants would use a dynamic consent mechanism to update their preferences when they change.

To begin to address the first of these empirical questions, we took advantage of an opportunity to examine the stability of a particular donor preference expressed at the time donors were enrolled in a large DNA biobank. Our objective was to examine the stability of this preference over time, contributing empirical data on the potential utility of a dynamic consent model for biobank participation.

\section{Materials and methods}

\section{Participants}

The sample was drawn from the Mayo Clinic biobank $(n=$ 50,702 at the time of the survey). Informed consent for biobank participation had been obtained in-person for a small percentage of early participants $(n=1302,2.6 \%)$, with the majority of participants enrolling via a mailed consent form accompanied by an informational brochure [10]. The consent form signed by biobank participants at the time of enrollment provided an option for participants to restrict posthumous availability of their biospecimens to the donor's legal next-of-kin (see Fig. 1). This preference was solicited from participants during the biobank enrollment process based on a recommendation by the community advisory board serving the Mayo Clinic biobank. No education or ancillary information regarding this opt-out opportunity was provided in the context of biobank enrollment (i.e., in the consent form or in the informational brochure provided in the mailed enrollment opportunity). A small subset of biobank enrollees ( $n=1314,2 \%$ at the time of the study) selected the option to restrict posthumous availability of their biospecimen to their legal next-of-kin. After screening this group to verify vital status, study eligibility, and mailing address, we identified a subset of 840 biobank donors whom we defined as "T1 restrictors." In addition to these individuals, 840 biobank enrollees who selected the option to permit posthumous availability of their sample by legal next-of-kin ("T1 permitters") were matched on age and sex to those from the biobank population who restricted posthumous biospecimen availability.

\section{Survey}

We designed a 66-item questionnaire containing both investigator-developed items and items from previously validated psychosocial instruments. At the beginning of the survey, we included a question which asked participants to indicate their current preference regarding the posthumous availability of their biospecimen to their next-of-kin. Since this preference was initially solicited from participants in the biobank consent form, we included the explanatory text from the consent form and reminded participants that they had previously indicated their preference when they enrolled in the biobank. No educational information was provided regarding this preference (i.e., nothing beyond information provided in Fig. 1), and care was taken to present the preference in the same manner as it was presented during biobank enrollment. The question was cast as a hypothetical choice (i.e., "If you were presented this option today...") so as not to indicate to participants that they were effectively modifying their biobank registration. To avoid influencing their current response, we did not remind participants of the specific preference they had provided previously. The survey then asked respondents to rate the strength of their current preference immediately after indicating whether they would choose to restrict or permit posthumous availability of their sample to their nextof-kin. Participants rated the strength of their current preference on a 3 point scale ("very strongly, somewhat strongly, not at all strongly"). 
Additional study-specific items included a list of nine statements addressing decision-making factors (e.g., "I believe my family might worry about genetic results they could learn if they had access to my sample"), followed by an "agree-unsure-disagree" response set. Participants were asked to indicate the level of importance (i.e., "not at all important, somewhat important, very important") of each of these factors and finally to identify one factor as a prevailing decision-making priority. A write-in option was provided to enable participants to record factors that were absent from our list but that influenced their decision to restrict or permit family access. Not all survey data are presented in this paper.

Surveys included the Self Concealment Scale (SCS) [11] and three subscales from the Family Environment Scale (FES) [12]. The SCS measures an individual's tendency to hide information from others. From the FES, we included three subscales-cohesion, expressiveness, and conflict-to examine relationships between these aspects of family functioning and preferences for posthumous sample availability. We hypothesized that an individual's tendency toward self-concealment (SCS), or family environments low on cohesion and expressiveness and high on conflict (FES), would be associated with a tendency to restrict posthumous availability of their sample to family members.

\section{Data collection}

The study was deemed exempt by the Mayo Clinic Institutional Review Board (\#15-000752). Biobank participants were mailed a copy of the survey along with an explanatory cover letter and a postage-paid return envelope, with one repeat mailing to nonresponders. Returned surveys were processed by research support staff who followed established procedures and methods to enhance data quality, including response tracking and double data entry.

\section{Data analysis}

Participant responses to the survey question about posthumous availability of their biospecimen were compared with their response to the same question elicited at the time of their initial enrollment in the biobank. This exercise identified four subgroups for analysis: (A) participants who permitted availability both at biobank enrollment (T1) and on the survey (T2), (B) participants who restricted availability at both $\mathrm{T} 1$ and $\mathrm{T} 2,(\mathrm{C})$ participants who permitted availability at $\mathrm{T} 1$, but at $\mathrm{T} 2$ changed their preference to restrict availability, and (D) participants who restricted sample availability at $\mathrm{T} 1$, but at $\mathrm{T} 2$ changed their preference to permit availability. Groups A and B were considered "consistent" in their preference (i.e., "consistent permitters"

\begin{tabular}{|l|c|c|c|}
\hline & \multicolumn{2}{|c|}{$\begin{array}{c}\text { Preference for posthumous } \\
\text { sample availability }\end{array}$} \\
\cline { 2 - 4 } & $\begin{array}{c}\text { At Consent } \\
\text { (T1) }\end{array}$ & & $\begin{array}{c}\text { On Survey } \\
\text { (T2) }\end{array}$ \\
\hline Descriptor & Permit & $\longrightarrow$ Permit \\
\hline Consistent Permitters & Restrict & $\longrightarrow$ & Restrict \\
\hline Inconsistent Permitters & Permit & $\longrightarrow$ & Restrict \\
\hline Inconsistent Restrictors & Restrict & $\longrightarrow$ & Permit \\
\hline
\end{tabular}

Fig. 2 Survey respondent subgroups. The following descriptors are used throughout the paper to refer to the four groupings of participants based on responses in the consent form and on the survey to the question about the posthumous availability of participants' biospecimens.

and "consistent restrictors"), while groups C and D were considered "inconsistent" in their preference (i.e., "inconsistent permitters" and "inconsistent restrictors" with respect to their initial preference indicated at biobank consent). Figure 2 illustrates this fourfold division of participants based on participants' preferences elicited at $\mathrm{T} 1$ and $\mathrm{T} 2$.

Descriptive statistics were calculated for demographic variables, for factors influencing participants' preference for biospecimen availability, for the SCS and FES scales, and for the access preference variable. Chi-square and $t$ tests were run to compare the distribution of responses across key demographic variables for participants with static and fluid preferences and by access preference. Statistical software packages SAS 9.4 (SAS institute Inc. Cary NC) and R 3.1 (R Foundation for Statistical Computing, Vienna, Austria) were used for data analysis.

\section{Results}

A total of 1207 individuals returned a survey for a response rate of $71.9 \%$. Forty-three of these responses were refusals to participate, resulting in 1164 records for analysis $(69.3 \%$ of the original sample). Responses received were evenly distributed between participants who restricted posthumous availability of their sample at the time of biobank consent (T1) $(n=584,50.2 \%)$ and those who permitted availability at $\mathrm{T} 1(n=580,49.8 \%)$.

Table 1 compares demographic variables of all participants in the Mayo Clinic Biobank who permitted availability of their biobank sample at T1 $(N=49,388)$ with those who restricted availability of their biobank sample at $\mathrm{T} 1(N=1314)$. All differences reported are statistically significant. More permitters than restrictors were married, and fewer permitters were single or 
Table 1 Comparison of demographic characteristics of all biobank participants $^{\mathrm{a}}$ by choice, at time of consent, to restrict or permit posthumous availability of biospecimens.

\begin{tabular}{llll}
\hline Characteristic $^{\mathrm{b}}$ & $\begin{array}{c}\text { Permitters }^{\mathrm{d}} \\
(N=49,388)\end{array}$ & $\begin{array}{c}\text { Restrictors }^{\mathrm{c}} \\
(N=1314)\end{array}$ & $p$ \\
\hline Age & & & $<0.000$ \\
Mean (SD) & $62.4(15.6)$ & $66.3(14.3)$ & \\
Median, range & 64.4, & 67.8, & \\
& $18.2-103.5$ & $19.8-98.3$ &
\end{tabular}

Time since biobank

enrollment

$\begin{array}{lll}\leq 1 \text { year } & 9402(19.0 \%) & 313(23.8 \%) \\ >1 \text { year } & 39,986 & 1001(76.2 \%) \\ & (81.0 \%) & \end{array}$

Last invited to participate

0.0176

in research

Never invited
$\leq 1$ year
$1-3$ years
$>3$ years
Gender
Male
Female
Race
White
Non-White
Education
Less than high school
diploma
High school
diploma or GED
Some college
College graduate
Graduate education

$\begin{array}{ll}39935(80.9 \%) & 1078(82.0 \%) \\ 2173(4.4 \%) & 74(5.6 \%) \\ 3037(6.1 \%) & 63(4.8 \%) \\ 4243(8.6 \%) & 99(7.5 \%) \\ & \\ 20276(41.1 \%) & 422(32.1 \%) \\ 29112(58.9 \%) & 892(67.9 \%) \\ 44900(92.4 \%) & 1178(90.7 \%) \\ 3695(7.6 \%) & 121(9.3 \%) \\ & \\ 829(1.7 \%) & 29(2.3 \%) \\ 6963(14.6 \%) & 258(20.4 \%) \\ 15454(32.3 \%) & 446(35.2 \%) \\ 12281(25.7 \%) & 272(21.5 \%) \\ 12295(25.7 \%) & 262(20.7 \%)\end{array}$

Marital status at time of consent

\begin{tabular}{lll} 
Single & $3448(7.3 \%)$ & $131(10.3 \%)$ \\
Married & $37848(79.8 \%)$ & $904(71.3 \%)$ \\
$\begin{array}{l}\text { Separate/divorced/ } \\
\text { widowed }\end{array}$ & $6161(13.0 \%)$ & $232(18.3 \%)$ \\
\hline
\end{tabular}

${ }^{\mathrm{a}}$ All participants at the time the survey was fielded (July, 23, 2015).

${ }^{\mathrm{b}}$ Unless otherwise noted, the format for all values is $N(\%)$.

${ }^{\mathrm{c}}$ This represents the study sample frame from which we derived our sample of individuals restricting access at time of consent.

${ }^{\mathrm{d}}$ This represents the study sample frame from which we derived our matched controls.

separated/divorced/widowed $(p<0.0001)$. More women than men restricted access, and permitters were slightly more educated ( $p<0.0001$ for both).

Table 2 compares demographic characteristics of survey respondents who permitted posthumous availability of their biobank sample at $\mathrm{T} 1$ irrespective of their $\mathrm{T} 2$ preference
( $n=580)$ with two groups: (1) all respondents who restricted availability at T1 irrespective of T2 preference $(n=584)$ and (2) respondents who were "consistent restrictors" $(n=$ 136) - those who restricted sample availability at $\mathrm{T} 1$ and $\mathrm{T} 2$. Those who permitted availability of their sample at $\mathrm{T} 1$ differed from those who restricted availability at $\mathrm{T} 1$ and from consistent restrictors in that more respondents who permitted access at $\mathrm{T} 1$ were members of the biobank for $>1$ year. In addition, more respondents who permitted access at $\mathrm{T} 1$ had never been invited to research requiring reconsent $(p<$ 0.0001 for all comparisons). More consistent restrictors were single and separated/divorced/widowed than those who permitted sample availability at $\mathrm{T} 1 \quad(p=0.003)$. Fewer consistent restrictors $(56.8 \%)$ were survived by siblings than those who permitted sample availability at $\mathrm{T} 1(88.5 \%, p=$ $0.04)$. Fewer restrictors at $\mathrm{T} 1$ and consistent restrictors (77.5\% and $56.8 \%$, respectively) were survived by children than participants who permitted availability at $\mathrm{T} 1(88.5 \%$, $p<0.0001$ for both).

Figure 3 describes the stability of participants' preferences for posthumous sample availability as reported at the time of consent (T1) and time of the survey (T2). Participants whose preference at time of the survey agreed with their preference at time of consent are compared with participants whose preference changed between time points. Importantly, over $40 \%$ of the entire sample had inconsistent preferences between T1 and T2. Further, 94\% of those with inconsistent preferences shifted from a $\mathrm{T} 1$ preference to restrict posthumous availability of their sample to a $\mathrm{T} 2$ preference to make the sample available to their legal nextof-kin. $76.7 \%$ of those who restricted availability at $\mathrm{T} 1$ chose to permit availability at T2, leaving only $136(23.3 \%)$ "consistent restrictors" who indicated a restrictive preference at both T1 and T2. Since those who restricted availability at $\mathrm{T} 1$ already represented a small subset of the entire biobank ( 2\%), the residual percentage of "consistent restrictors" in the entire biobank is remarkably small.

Consistent permitters indicated a "very strongly" held preference more frequently (68.9\%) than consistent restrictors $(47.4 \%, p<0.0001)$. Participants with inconsistent preferences indicated a "very strongly" held preference more frequently than consistent restrictors, but less frequently than consistent permitters. Over $70 \%$ of $\mathrm{T} 1$ restrictors who were members of the biobank for less than 1 year at the time of the survey indicated a preference change at $\mathrm{T} 2$.

Table 3 examines the preference stability of the groups shown in Fig. 3 across demographic variables. Inconsistent permitters who chose to restrict access at $\mathrm{T} 2$ were older $($ mean $=71.5$ years, $S D=12.4)$ than consistent permitters $($ mean $=66.1$ years, $\mathrm{SD}=13.1, p=0.0343$ ). One hundred percent of inconsistent restrictors who permitted access at $\mathrm{T} 2$ were survived by at least one blood 
Table 2 Demographic characteristics of survey respondents who permitted access to their sample at the time of initial consent, compared with respondents who restricted sample access at $\mathrm{T}_{1}$ and at both $\mathrm{T}_{1}$ and $\mathrm{T}_{2}$.

\begin{tabular}{|c|c|c|c|c|c|}
\hline \multirow[t]{2}{*}{ Characteristic $^{\mathrm{a}}$} & \multirow{2}{*}{$\begin{array}{l}\text { All permitters } \\
\mathrm{T}_{1}, N=580^{\mathrm{b}}\end{array}$} & \multicolumn{4}{|l|}{ Restrictors } \\
\hline & & $\begin{array}{l}\text { All } \mathrm{T}_{1} \\
N=584^{\mathrm{c}}\end{array}$ & $p$ & $\begin{array}{l}\mathrm{T}_{1} \text { and } \mathrm{T}_{2} \\
N=136^{\mathrm{d}}\end{array}$ & $p$ \\
\hline \multicolumn{2}{|l|}{ Age } & \multicolumn{3}{|c|}{0.9561} & 0.2348 \\
\hline Mean (SD) & $66.4(13.1)$ & $66.4(13.5)$ & & $64.6(15.2)$ & \\
\hline Median, range & $68.0,20-97$ & $67.8,20-97$ & & $66.4,23-95$ & \\
\hline \multicolumn{2}{|l|}{ Time since biobank enrollment } & & $<0.0001$ & & 0.0001 \\
\hline$\leq 1$ year & $158(27.2)$ & 227 (38.9) & & $61(44.9)$ & \\
\hline$>1$ year & $422(72.8)$ & $357(61.1)$ & & $75(55.1)$ & \\
\hline \multicolumn{2}{|l|}{$\begin{array}{l}\text { Last invited to participate in } \\
\text { research }\end{array}$} & & $<0.0001$ & & $<0.0001$ \\
\hline Never invited & $550(94.8)$ & $492(84.2)$ & & $113(83.1)$ & \\
\hline$\leq 1$ year & $2(0.3)$ & $54(9.2)$ & & $17(12.5)$ & \\
\hline $1-3$ years & $11(1.9)$ & $12(2.1)$ & & $1(0.7)$ & \\
\hline$>3$ years & $17(2.9)$ & $26(4.5)$ & & $5(3.7)$ & \\
\hline \multicolumn{2}{|l|}{ Gender } & & 0.4266 & & 0.5819 \\
\hline Male & $182(31.4)$ & $196(33.6)$ & & $46(33.8)$ & \\
\hline Female & $398(68.6)$ & $388(66.4)$ & & $90(66.2)$ & \\
\hline \multicolumn{2}{|l|}{ Race } & & 0.6954 & & 0.4511 \\
\hline White & $534(92.4)$ & $535(91.8)$ & & $123(90.4)$ & \\
\hline Non-White & $44(7.6)$ & $48(8.2)$ & & $13(9.6)$ & \\
\hline \multicolumn{2}{|l|}{ Education } & & 0.2901 & & 0.1582 \\
\hline $\begin{array}{l}\text { Less than high school } \\
\text { diploma }\end{array}$ & $8(1.4)$ & $9(1.6)$ & & $5(3.7)$ & \\
\hline High school diploma or GED & $91(15.9)$ & $113(19.8)$ & & $29(21.3)$ & \\
\hline Some college & $184(32.1)$ & $188(33.0)$ & & $43(31.6)$ & \\
\hline College graduate & $129(22.5)$ & $126(22.1)$ & & $29(21.3)$ & \\
\hline Graduate education & $161(28.1)$ & $134(23.5)$ & & $30(22.1)$ & \\
\hline \multicolumn{2}{|l|}{ Marital status at time of consent } & & 0.0849 & & 0.0030 \\
\hline Single & $32(5.6)$ & $48(8.3)$ & & $17(12.6)$ & \\
\hline Married & $457(79.5)$ & $430(74.5)$ & & $91(67.4)$ & \\
\hline Separate/divorced/widowed & $86(15.0)$ & $99(17.2)$ & & $27(20.0)$ & \\
\hline \multicolumn{6}{|l|}{ Survived by } \\
\hline Anyone & $573(98.8)$ & $580(99.3)$ & 0.3574 & $132(97.1)$ & 0.1389 \\
\hline Grandparents & $25(4.4)$ & $32(5.5)$ & 0.3733 & $10(7.6)$ & 0.1305 \\
\hline Parents & $189(33.2)$ & $182(31.5)$ & 0.5452 & $46(34.8)$ & 0.7107 \\
\hline Siblings & $507(88.5)$ & $504(87.0)$ & 0.4574 & $108(81.8)$ & 0.0386 \\
\hline Children & $507(88.5)$ & 449 (77.5) & $<0.0001$ & $75(56.8)$ & $<0.0001$ \\
\hline
\end{tabular}

${ }^{a}$ Unless otherwise noted, the format for all values is $N(\%)$.

${ }^{\mathrm{b}}$ Survey respondents who permitted next-of-kin access to their biobank sample at consent (T1).

${ }^{\mathrm{c}}$ Survey respondents who restricted next-of-kin access to their biobank sample at consent (T1).

${ }^{\mathrm{d}}$ Survey respondents who restricted next-of-kin access at consent and at the time of survey (T1 and T2). relative, compared with $97.1 \%$ of consistent restrictors ( $p=0.0028) .83 .7 \%$ of inconsistent restrictors who permitted access at T2 compared with just $56.8 \%$ of consistent restrictors were survived by at least one child $(p<$ $0.0001)$. These demographic differences may highlight the role that family composition plays in decisions about posthumous sample availability.

\section{Discussion}

Results from our study suggest that biobank participants' preferences regarding sample access may change over time, providing some initial empirical support for the claim that biobank participants may have reason to utilize a dynamic consent mechanism to manage their participation. Kaye 


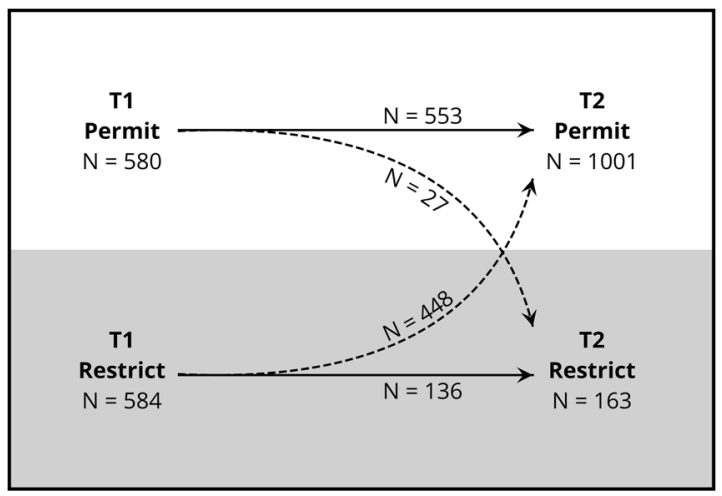

Fig. 3 Stability of participants' next-of-kin access preference. Stability of participants' next-of-kin access preference between $\mathrm{T}_{1}$ (time of biobank consent) and $\mathrm{T}_{2}$ (time of survey completion), $n=$ 1164. Solid arrows indicate participants whose access preferences were the same at $T_{1}$ and $T_{2}$. Dotted lines indicate participants whose access preference changed between $T_{1}$ and $T_{2}$.

et al. have proposed a dynamic consent model as a method of biobank stewardship [1]. The case for this model, while not predicated on the assumption that donor preferences change over time, is strengthened by empirical data demonstrating that biobank donors may have reasons to modify their sample use preferences.

Few studies to date have focused on the opinions and preferences of individuals who have been enrolled in a biobank for several years [13]. To the best of our knowledge, no prior studies have explored whether the preferences of biobank participants remain consistent in the months and years after donors provided their initial consent. Although recent empirical work in biobanking ethics has moved beyond the question of whether broad consent is ethically acceptable, this literature has focused largely on the views and opinions of individuals who are being actively recruited into a biobank [14, 15]. Several studies have focused on consent for biobank participation with the recognition that biobank participation requires a unique level of participant trust. Some of these studies have sought to make informed consent for biobanking more effective [16-18] and less cognitively burdensome [19-21]. Other studies have uncovered potential barriers (e.g., privacy, confidentiality) to biobank participation [22, 23], clarified patient preferences for different models of consent [24-28], and examined the impact of "non-welfare interests" on decisions to donate biological materials [29]. van Zon et al. [30] identified 25 studies focused on methods or strategies for improving biobank participation. While all of these considerations are important and represent a shift in focus from more abstract concerns about the curation of biospecimens to studies examining the actual experience and preferences of the biospecimen donors, few studies have examined the stability of donor preferences regarding sample use years after their decision to enroll in a DNA biobank.
Our survey identified a degree of inconsistency between initially stated donor preferences regarding posthumous sample availability and donor preferences elicited later (at the time of our survey). While it is reasonable to assume that many biobank participants may not recall answering the question about posthumous availability of their biobank sample when they consented to enroll in the biobank, we did not expect such a high proportion $(>40 \%)$ of survey respondents to indicate a current preference that did not align with the preference they indicated at the time of enrollment. These shifting preferences may be attributable to weak initial preferences that are subject to change. Our data on strength of preference suggest that some individuals who chose to restrict availability at T1 may have simply felt that this option was a reasonable "default choice," but did not have a strong opinion about this choice. Shifting preferences may be related to changes in life circumstances or changes in family composition or relationships. Changes in preference may also reflect shifting views on the importance of biomedical research or broader attitudes about the importance of protecting data privacy. Many of the inconsistent restrictors were members of the biobank for $>1$ year. More time between preference specification points provides greater opportunity for shifts in personal views and changes in family composition and dynamics.

Unfortunately, our data provide limited insights as to what may be driving these changes of preference over time. For instance, data on participants' age, marital status at time of consent, family cohesion, and vital status of blood relatives suggest that familial changes may be a factor as participants form their preferences around sharing and access. A strong majority (94\%) of those who changed preference between $\mathrm{T} 1$ and $\mathrm{T} 2$ were inconsistent restrictors who, at $\mathrm{T} 2$, shifted in their preference to permit posthumous availability of their biospecimens (with just $6 \%$ of participants shifting from a $\mathrm{T} 1$ preference of permitting sample availability to a $\mathrm{T} 2$ preference of restricting availability to their legal nextof-kin). There are a number of possible explanations for this finding. Some participants may not have reflected on the potential implications of restricting posthumous availability when they were initially approached and were asked to participate in the biobank, but when presented with the option a second time, these individuals may have considered this topic more thoroughly. Similarly, some participants may have misunderstood the question at the time of consent and although their preference appeared to change, their authentic preference remained consistent. Still others may have acquired a comfort with biobank participation since their enrollment, perhaps as a result of receiving updates through biobank newsletters and other communication. When asked about their preferences regarding sample availability a second time, some donors may have come to appreciate the potential benefits of permitting their 
Table 3 Demographic comparison of participants with consistent vs. inconsistent preferences, grouped by preference indicated at consent.

\begin{tabular}{|c|c|c|c|c|c|c|}
\hline \multirow[t]{2}{*}{ Characteristic $^{\mathrm{a}}$} & \multirow{2}{*}{$\begin{array}{l}\text { Consistent permitters } \\
\text { Permit } \rightarrow \text { Permit } \\
(N=553)\end{array}$} & \multicolumn{2}{|l|}{ Inconsistent permitters } & \multirow{2}{*}{$\begin{array}{l}\text { Consistent } \\
\text { restrictors } \\
\text { Restrict } \rightarrow \text { Restrict } \\
(N=136)\end{array}$} & \multicolumn{2}{|c|}{ Inconsistent restrictors } \\
\hline & & $\begin{array}{l}\text { Permit } \rightarrow \text { Restrict } \\
(N=27)\end{array}$ & $p$ & & $\begin{array}{l}\text { Restrict } \rightarrow \text { Permit } \\
(N=448)\end{array}$ & $p$ \\
\hline Age & & & 0.0343 & & & 0.1241 \\
\hline Mean (SD) & $66.1(13.1)$ & $71.5(12.4)$ & & $64.9(15.2)$ & $67.0(12.9)$ & \\
\hline Median, range & $67.8(20-97)$ & $73.8(38-95)$ & & $66.4(23-95)$ & $68.3(20-97)$ & \\
\hline Time since biobank enrollment & & & 0.4665 & & & 0.1022 \\
\hline$\leq 1$ year & $149(26.9)$ & $9(33.3)$ & & $61(44.9)$ & $166(37.1)$ & \\
\hline$>1$ year & $404(73.1)$ & $18(66.7)$ & & $75(55.1)$ & $282(62.9)$ & \\
\hline Last invited to a research study & & & 0.1471 & & & 0.3266 \\
\hline Never invited & $524(94.8)$ & $26(96.3)$ & & $113(83.1)$ & $379(84.6)$ & \\
\hline$\leq 1$ year & $1(0.2)$ & $1(3.7)$ & & $17(12.5)$ & $37(8.3)$ & \\
\hline $1-3$ years & $11(2.0)$ & $0(0)$ & & $1(0.7)$ & $11(2.5)$ & \\
\hline$>3$ years & $17(3.1)$ & $0(0)$ & & $5(3.7)$ & $21(4.7)$ & \\
\hline Gender & & & 0.5165 & & & 0.9411 \\
\hline Male & $172(31.1)$ & $10(37.0)$ & & $46(33.8)$ & $150(33.5)$ & \\
\hline Female & $381(68.9)$ & $17(63.0)$ & & $90(66.2)$ & $298(66.5)$ & \\
\hline Race & & & 0.4506 & & & 0.5927 \\
\hline White & $510(92.6)$ & $24(88.9)$ & & $123(90.4)$ & $412(92.2)$ & \\
\hline Non-White & $41(7.4)$ & $3(11.1)$ & & $13(9.6)$ & $35(7.8)$ & \\
\hline Education & & & 0.2810 & & & 0.2811 \\
\hline $\begin{array}{l}\text { Less than high school } \\
\text { diploma }\end{array}$ & $7(1.3)$ & $1(3.7)$ & & $5(3.7)$ & $4(0.9)$ & \\
\hline High school diploma or GED & $88(16.1)$ & $3(11.1)$ & & $29(21.3)$ & $84(19.4)$ & \\
\hline Some college & $174(31.9)$ & $10(37.0)$ & & $43(31.6)$ & $145(33.4)$ & \\
\hline College graduate & $126(23.1)$ & $3(11.1)$ & & $29(21.3)$ & $97(22.4)$ & \\
\hline Graduate education & $151(27.7)$ & $10(37.0)$ & & $30(22.1)$ & $104(24.0)$ & \\
\hline Marital status at time of consent & & & 0.1368 & & & 0.0538 \\
\hline Married & $440(80.1)$ & $17(65.4)$ & & $91(67.4)$ & $339(76.7)$ & \\
\hline Single & $79(14.4)$ & 7 (26.9) & & $17(12.6)$ & $72(16.3)$ & \\
\hline Separate/divorced/widowed & $79(14.4)$ & $2(7.7)$ & & $27(20.0)$ & $31(7.0)$ & \\
\hline \multicolumn{7}{|l|}{ Survived by } \\
\hline Anyone & 551 (99.6) & $22(81.5)$ & $<0.0001$ & $132(97.1)$ & $448(100.0)$ & 0.0028 \\
\hline Grandparents & $25(4.6)$ & $0(0.0)$ & 0.6161 & $10(7.6)$ & $22(4.9)$ & 0.2775 \\
\hline Parents & $185(33.8)$ & $4(18.2)$ & 0.1668 & $46(34.8)$ & $136(30.5)$ & 0.3397 \\
\hline Siblings & $486(88.2)$ & $21(95.5)$ & 0.4964 & $108(81.8)$ & 396 (88.6) & 0.0542 \\
\hline Children & $490(88.9)$ & $19(86.4)$ & 0.7264 & $75(56.8)$ & $374(83.7)$ & $<0.0001$ \\
\hline
\end{tabular}

${ }^{a}$ Unless otherwise noted, the format for all values is $N(\%)$.

biospecimen to be available to blood relatives. While it is impossible from our data to ascertain the motivations behind these preference changes, these, and other explanations seem plausible.

Our data also suggest that preferences collected during the initial consent process do not dependably predict longterm opinions of biobank participants. Studies have found that a high percentage of those approached about biobank membership have never heard of a biobank [31, 32]. This suggests that many individuals who enroll in biobanks may be expressing preferences at the same moment in time in which they are learning what a biobank is and what their participation entails. Soliciting broad consent in this context presumes that donors are capable of quickly integrating information they receive at the time of consent into their own personalized risk-benefit calculus and making an informed decision that will be consistent with their preferences over time. Whether or not this is an appropriate presumption, there is reason to believe that preference adjustments made in the months or years after enrollment 
are either the product of greater reflection on the implications of permitting sample availability or are a response to changing life circumstances.

The practice of not regularly revisiting and collecting the current preferences of biobank donors may result in missed opportunities for responsible sample stewardship. Biobank donors are part of complex social and familial networks. Shifts in donor preferences regarding sample uses may reflect changes in life circumstances, changes in familial relationships, or changes resulting from significant life experiences such as illness or the death of a family member. Failing to acknowledge and seek to accommodate these changing life experiences and their impact on personal preferences might be seen as a type of disrespectful treatment. In addition, failing to revisit donor preferences over time may be a missed opportunity to strengthen the trust of biobank donors by acknowledging their gift and reaffirming a commitment to using it in a manner that is consistent with their preferences. Donors need not express a preference change in order to appreciate good-faith efforts to accommodate evolving donor preferences.

The consent form used in the biobank we examined provided a single option regarding posthumous availability of donated biospecimens and did not solicit other specific preferences from sample donors. Other potentially fluid preferences might include preferences regarding the reporting of individual research results, the communication of aggregated study findings, or the notification of sample sharing with specific researchers. Future research could seek to clarify whether these and other specific preferences are more or less fluid as a result of changing family structures or evolving life circumstances. The specific preference we examined in this survey study is highly unique in that it requires individuals to reflect on whether the availability of a donated biospecimen would have potential benefits for family members or pose risks to one's family or one's personal reputation after death. Donors must weigh these abstract risks and benefits and choose which option they prefer. This decision may be cognitively burdensome because it requires donors to conceptualize the scope of their sharing and the potential implications of posthumous specimen availability. Other preferences, such as return-ofresults preferences, may not be as burdensome to evaluate or have as complex implications for others, and thus may not be as likely to change over time. It is unclear which donor preferences regarding sample use are most likely to change over time.

The greatest challenge against implementing preferencesensitive mechanisms like dynamic consent for ongoing biospecimen management is the cost of implementation and maintenance. Biobanks containing thousands or tens of thousands of samples might struggle to transition from their current sample management procedures to some sort of dynamic consent approach. In some cases, additional IRB requirements may apply, including expectations that patients be recontacted and informed about these changes. While difficult to quantify, the level of trust instilled by such a system-both among those who modify their preferences and among those who are simply grateful that such an option exists-is a strong argument for considering the adoption of a dynamic consent model.

As one of the first studies of the stability of biobank donor preferences over time, our study has multiple limitations. First, participants in the biobank we examined were predominantly white, over 50 years of age, and well educated, as illustrated by demographic data in Table 2. As a result, it would be inappropriate to generalize our findings (e.g., the frequency of preference change) to more diverse populations or other research settings.

Second, as noted above, the question about posthumous sample availability is unusual, and it is possible that those who restricted access at $\mathrm{T} 1$ may have misunderstood the question when initially asked. This particular preference was the only preference solicited during the biobank enrollment process (see Fig. 1), and preferences around other, less abstract options may have been more or less stable between time points. In scenarios like this, where the approach to ascertaining a specific preference may have been deficient for some participants, dynamic consent could provide opportunity for corrections. The consent form provided no explanation of the potential value of restricting or permitting access to next-of-kin, and some individuals may have defaulted to a preference of greater control simply because they were not aware of any reason to do otherwise.

Third, our study did not approximate a simulation of a dynamic consent mechanism, which would facilitate preference modification but would not actively prompt participants to reevaluate their preferences as we did in our survey study. Further, had we reminded participants of their previous preference (as would be the case in a dynamic consent model), we might not have seen as much preference change as we observed in our study because some participants may have deferred to their previously expressed preference. Therefore, while our data suggest that biobank donor preferences may be fluid when elicited at two time points, they do not suggest whether preference modification mechanisms would be utilized by participants. Our data also do not speak to the comparative impact on preference stability of "opt-in" preference elicitation compared to "optout" preference elicitation.

Our study was also limited by a lack of a several important repeated measures that might clarify contributors to preference instability. Specifically, we lacked data at T1 (biobank enrollment) regarding the "survived-by" status of biobank participants. We collected this data at T2 (survey) but could not examine changes in family survivorship 
dynamics between time points. Similarly, we collected marital status at $\mathrm{T} 1$, but did not collect marital status at T2. This represents a missed opportunity to examine the impact of changes in family structure on biobank participant preferences. Our dataset also lacked information on changing health status between $\mathrm{T} 1$ and $\mathrm{T} 2$, as well as information on self-reported positive and negative research experiences. We did not anticipate the potential value of these measures during survey development because they were either (1) not feasible to collect, or (2) attained meaning only after we observed considerable instability of donor preferences in our sample.

Despite these limitations, it is clear that our data challenge assumptions about the stability of biobank donor preferences over time. Future research should examine potential explanations of this instability in more diverse biobank populations and with respect to a broader array of preferences regarding sample use and distribution. Future research should also examine more robustly whether or not modern family dynamics lie behind much of the sample use concerns and preferences of prospective and current biobank donors. Such findings would greatly enhance not only the debate about the merits of dynamic consent but also more high level considerations in biobank stewardship.

\section{Conclusion}

Is dynamic consent a solution to the potential shortcomings of traditional, broad consent for biobank research? While that is still a normative question, the answer must not neglect empirical realities, such as the extent to which the initial preferences indicated by sample donors actually change in substantive ways over time. More fully characterizing the stability of biobank donor preferences is a necessary first step in making an empirical case for the utility of dynamic consent for honoring participants' current sample use preferences. To the extent that biobanks curate not only biospecimens and health histories, but also public trust, sensitivity to evolving donor preferences is essential to the long-term success of these critical research resources.

Acknowledgements The authors wish to acknowledge the contributions of the late Carmen Radecki Breitkopf, Ph.D. to this work. This study was funded by the Mayo Clinic Center for Individualized Medicine and the Mayo Clinic Biobank.

Funding This study was funded by the Center for Individualized Medicine, Mayo Clinic.

\section{Compliance with ethical standards}

Conflict of interest The authors declare that they have no conflict of interest.
Publisher's note Springer Nature remains neutral with regard to jurisdictional claims in published maps and institutional affiliations.

\section{References}

1. Kaye J, Whitley EA, Lund D, Morrison M, Teare H, Melham K. Dynamic consent: a patient interface for twenty-first century research networks. Eur J Hum Genet. 2015;23:141-6.

2. Budin-Ljøsne I, Teare HJA, Kaye J, Beck S, Bentzen HB, Caenazzo L, et al. Dynamic consent: a potential solution to some of the challenges of modern biomedical research. BMC Med Ethics. 2017;18:4.

3. Sundby A, Boolsen MW, Burgdorf KS, Ullum H, Hansen TF, Middleton A, et al. The preferences of potential stakeholders in psychiatric genomic research regarding consent procedures and information delivery. Eur Psychiatry. 2019;55:29-35.

4. Denny JC, Rutter JL, Goldstein DB, Philippakis A, Smoller JW, Jenkins G, et al. The "All of Us" research Program. N Engl J Med. 2019;381:668-76.

5. Bledsoe MJ. Ethical legal and social issues of biobanking: past, present, and future. Biopreservation Biobanking. 2017;15:142-7.

6. Caulfield T, Murdoch B. Genes, cells, and biobanks: yes, there's still a consent problem. PLoS Biol. 2017;15:e2002654.

7. Chalmers D, Nicol D, Kaye J, Bell J, Campbell AV, Ho CWL, et al. Has the biobank bubble burst? Withstanding the challenges for sustainable biobanking in the digital era Donna Dickenson, Sandra Soo-Jin Lee, and Michael Morrison. BMC Med Ethics. 2016;17:39.

8. Critchley C, Nicol D, McWhirter R. Identifying public expectations of genetic biobanks. Public Underst Sci. 2017;26:671-87.

9. D'Abramo F, Schildmann J, Vollmann J. Research participants' perceptions and views on consent for biobank research: a review of empirical data and ethical analysis. BMC Med Ethics. 2015;16:60.

10. Olson JE, Ryu E, Johnson KJ, Koenig BA, Maschke KJ, Morrisette JA, et al. The Mayo Clinic Biobank: a building block for individualized medicine. Mayo Clin Proc. 2013;88:952-62.

11. Larson DG, Chastain RL. Self-concealment: conceptualization, measurement, and health implications. J Soc Clin Psychol. 1990;9:439-55.

12. Moos RH. Family environment scale. Palo Alto, Calif.: Consulting Psychologists Pr.; 1974.

13. Warner TD, Weil CJ, Andry C, Degenholtz HB, Parker L, Carithers LJ, et al. Broad consent for research on biospecimens: the views of actual donors at four U.S. medical centers. J Empir Res Hum Res Ethics. 2018;13:115-24.

14. Murphy J, Scott J, Kaufman D, Geller G, LeRoy L, Hudson K. Public perspectives on informed consent for biobanking. Am J Public Health. 2009;99:2128-34.

15. Brothers KB, Morrison DR, Clayton EW. Two large-scale surveys on community attitudes toward an opt-out biobank. Am J Med Genet A. 2011;155a:2982-90.

16. Beskow LM, Dombeck CB, Thompson CP, Watson-Ormond JK, Weinfurt KP. Informed consent for biobanking: consensus-based guidelines for adequate comprehension. Genet Med. 2015;17:226-33.

17. Cervo S, Rovina J, Talamini R, Perin T, Canzonieri V, De Paoli P, et al. An effective multisource informed consent procedure for research and clinical practice: an observational study of patient understanding and awareness of their roles as research stakeholders in a cancer biobank. BMC Med Ethics. 2013;14:30.

18. Beskow LM, Lin L, Dombeck CB, Gao E, Weinfurt KP. Improving biobank consent comprehension: a national 
randomized survey to assess the effect of a simplified form and review/retest intervention. Genet Med. 2017;19:505-12.

19. Simon CM, Klein DW, Schartz HA. Interactive multimedia consent for biobanking: a randomized trial. Genet Med. 2016;18:57-64.

20. Simon CM, Klein DW, Schartz HA. Traditional and electronic informed consent for biobanking: a survey of U.S. biobanks. Biopreservation Biobanking. 2014;12:423-9.

21. Beskow LM, Friedman JY, Hardy NC, Lin L, Weinfurt KP. Developing a simplified consent form for biobanking. PloS ONE. 2010;5:e13302.

22. Ridgeway JL, Han LC, Olson JE, Lackore KA, Koenig BA, Beebe TJ, et al. Potential bias in the bank: what distinguishes refusers, nonresponders and participants in a clinic-based biobank? Public Health Genom. 2013;16:118-26.

23. Banks E, Herbert N, Mather T, Rogers K, Jorm L. Characteristics of Australian cohort study participants who do and do not take up an additional invitation to join a long-term biobank: The 45 and Up Study. BMC Res Notes. 2012;5:655.

24. Kaufman D, Bollinger J, Dvoskin R, Scott J. Preferences for optin and opt-out enrollment and consent models in biobank research: a national survey of Veterans Administration patients. Genet Med. 2012;14:787-94.

25. Caulfield T, Rachul C, Nelson E. Biobanking, consent, and control: a survey of Albertans on key research ethics issues. Biopreservation Biobanking. 2012;10:433-8.
26. Ewing AT, Erby LA, Bollinger J, Tetteyfio E, Ricks-Santi LJ, Kaufman D. Demographic differences in willingness to provide broad and narrow consent for biobank research. Biopreservation Biobanking. 2015;13:98-106.

27. Joly Y, Dalpe G, So D, Birko S. Fair shares and sharing fairly: a survey of public views on open science, informed consent and participatory research in biobanking. PloS ONE. 2015;10: e0129893.

28. Master Z, Claudio JO, Rachul C, Wang JC, Minden MD, Caulfield $\mathrm{T}$. Cancer patient perceptions on the ethical and legal issues related to biobanking. BMC Med Genom. 2013;6:8.

29. De Vries RG, Tomlinson T, Kim HM, Krenz CD, Ryan KA, Lehpamer N, et al. The moral concerns of biobank donors: the effect of non-welfare interests on willingness to donate. Life Sci Soc Policy. 2016;12:3.

30. van Zon SK, Scholtens S, Reijneveld SA, Smidt N, Bultmann U. Active recruitment and limited participant-load related to high participation in large population-based biobank studies. J Clin Epidemiol. 2016;78:52-62.

31. Simon CM, L'Heureux J, Murray JC, Winokur P, Weiner G, Newbury E, et al. Active choice but not too active: public perspectives on biobank consent models. Genet Med. 2011;13:821-31.

32. Bossert S, Kahrass H, Strech D. The public's awareness of and attitude toward research biobanks - a regional German survey. Front Genet. 2018;9:190. 